Bull. Fac. Agric., Cairo Univ., 67: 19-30 (2016).

\title{
EFFECT OF INDOLE ACETIC ACID ON GROWTH AND YIELD \\ OF SOME NEW BARLEY VARIETIES AFTER CUTTING \\ UNDER NEW LAND CONDITIONS AT ISMAILIA
}

(Received: 13.12.2015)

\author{
By \\ M. Z. Shendy and M. A. Madkour* \\ Barley Research Department and *Crop Physiology Research Department, Field Crops Research \\ Institute, Agricultural Research. Center, Giza, Egypt.
}

\begin{abstract}
A two year field experiment was conducted at Ismailia indole acetic acid Agric. Res. Station during 2013/2014 and 2014/2015 seasons to study the effect of foliar spray with IAA after 20, 40 and twice at 20 and 40 days from cutting at 45 day plant age on growth and yield of three barley cultivars, Giza 133, Giza 134 and Giza 2000. Results indicated that all growth and yield characteristics under study of Giza 2000 had the highest values. The Data indicated that all characteristics decreased after cutting plants compared to non-cutting plants. Foliar spray of barley with indole acetic acid (IAA) after cutting either once at 20,40 and twice at 20 and 40 days significantly increased all growth and yield characteristics under study compared with the first treatment (cutting plants without foliar spray of IAA). Total carbohydrate content of grains increased by spraying plants with IAA compared to plants without foliar spray of IAA. Plants in control treatment gave the highest content of carbohydrates. Whereas, protein content of grains gradually decreased with foliar spray with IAA. Giza 2000 had the highest values for net benefit and marginal rate return percentage (MRR \%). Foliar spray of barley with indole acetic acid after cutting twice at 20 and 40 days had the highest values for net benefit and marginal rate return percentage.
\end{abstract}

Key words: Hordeum vulgare, growth hormones, barley cutting.

\section{INTRODUCTION}

Barley (Hordeum vulgare L.) is the main crop grown in rainfed areas of Egypt. It is adapted to grow satisfactorily under adverse conditions, i.e. drought, low fertility, saline soil, high or low temperature and moisture stress. Barley is the world's fourth most important cereal crop in terms of cultivated area. It is used for human consumption as well as animal feeding. Barley production area in Egypt is located in the North Coastal region and newly reclaimed lands (El-Bawab and Sandak, 2002). The effect of cutting or grazing on grain yield is influenced by environmental factors and plant growth regulators. Royo and Tribo (1997) reported that forage barley was cut when the first node was detectable and for grain was harvested at ripening. The biomass components at cutting were positively and significantly correlated with forage yield. The maximum number of living leaves per plant was reached between the beginning of jointing and booting. The number of living tillers per plant at anthesis was significantly higher. Grain filling in both cut and uncut harvesting treatments was mainly dependent on current photosynthesis after anthesis. Royo (1999) indicated that plant recovery after cutting for forage consumption was affected by environmental conditions, sowing date and plant stage at cutting. Delaying cutting for forage consumption caused a greater reduction in dry matter accumulation, flag area expansion and grain yield components. Yau (1999) found that early sowing without grazing gave the least straw and grain yield, but the least harvest index while normal sowing gave the highest grain and straw yield. Al-Satari et al. (2001) pointed out that a single clipping at the tillering stage produced the highest fresh and dry matter yield. The highest grain yield was obtained from unclipped plants, however clipping reduced grain yield. Sharma (2002) showed that in the first cutting, average fresh forage yield of barley was $15.39 \mathrm{t} \mathrm{ha}^{-1}$ and the average dry matter content was $17.08 \%$. Barley may be grown for forage, but due to the high 
biomass accumulation capacity during the early growth stage, a high forage yield may be expected from it. Yau and Yau (2003), reported that early planting with early clipping or grazing did not reduce grain and straw yield comparable with early or normal planting without clipping or grazing. Treatments were early planting with no clipping or grazing (ENG), early planting with early clipping or grazing (EG) and normal planting with no clipping or grazing (NNG). In comparison with the ENG and NNG treatments, the EG treatment did not reduce grain and straw yields. Thus, if farmers in semiarid areas plant their barley crop early and then allow greenstage grazing, they may gain a certain amount of nutrition forage without decreasing grain and straw production. Harsharn and Gill (1985) indicated that spraying barley with 100 ppm IAA at tillering and heading stages significantly increased the number of effective tillers plant ${ }^{-1}$, number of grains spike ${ }^{-1}, 1000$-grain weight, LAI and grain yield of barley compared with the control (water spray). Salem (1990) soaked seeds of barley in 50, 100 and 150 ppm IAA for $12 \mathrm{~h}$ before sowing in plots. Found that the highest concentration of IAA adversely affected fresh and dry weight. The decrease in carbohydrate and protein contents of control plant observed after 60 days, was reversed by treatment with growth regulator. Low concentration of IAA increased total carbohydrates. Barsoum (1994) concluded that grain yield was the highest by soaking barley seeds in 50 ppm IAA. Angela and Gray (2011) showed that plant growth and development require the integration of a variety of environmental and endogenous signals that together with the intrinsic genetic program, determine plant form. Central to this process are several growth regulators known as phytohormones. It is worthy enough to study the effect of some physiological factors, as the use of growth substances such as indole acetic acid (IAA) at different concentrations that may increase barley plant production. It is quite clear that endogenous and exogenous plant growth regulators play an important role in modifying and regulating many physiological processes in plants and these processes are greatly influenced by environmental conditions. Senthil et al. (2003) investigated the effect of IAA at $100 \mathrm{ppm}$ supplied as foliar spray at 35 and 60 days after sowing on some physiological aspects including total chlorophyll and soluble protein of soybean plants. They reported that all treatments increased the growth and yield characteristics of soybean and IAA treatments had the highest effect on the plant.

The present study aimed to investigate the effect of foliar spray with indole acetic acid (IAA) after cutting barley plants on growth and yield characteristics.

\section{MATERIALS AND METHODS}

A two-year field experiment was conducted at Ismailia Agric. Res. Station, A. R. C., Egypt during 2013/2014 and 2014/2015 seasons. The main objective of this study was to determine the effect of foliar spray of IAA after cutting barley plants at tillering stage on subsequent growth, yield and chemical contents of the grains. Each plot consisted of 8 rows, $3.5 \mathrm{~m}$ long and $20 \mathrm{~cm}$ apart. Potassium was added in the form of potassium sulphate $\left(48 \% \mathrm{~K}_{2} \mathrm{O}\right)$ at two equal doses at planting and 30 days later. Fertilizers were applied at the rate of $15 \mathrm{~kg} \mathrm{P}_{2} \mathrm{O}_{5}$ and $30 \mathrm{~kg}$ $\mathrm{N}$ fed ${ }^{-1}$. Phosphorus fertilizer was added in the form of calcium super phosphate $\left(15.5 \% \mathrm{P}_{2} \mathrm{O}_{5}\right)$ in one dose before planting. Nitrogen fertilizer was added in the form of ammonium nitrate $(33.5 \% \mathrm{~N})$ at six equal doses, at sowing and at 15, 30, 45, 60 and 75 days later. Cutting plants for the forage yield was done at 45 days after sowing. A split plot design with three replications was used. Three cultivars Giza 133, Giza 134 and Giza 2000 (sown on the $1^{\text {st }}$ of December in both seasons) in the main plots. The sub-plots included the control (no cut and without spray IAA) $\mathrm{T}_{0}$; cut plants without spray $\operatorname{IAA}\left(\mathrm{T}_{1}\right)$, cut plants and foliar spray with IAA at 20 days after cutting $\left(\mathrm{T}_{2}\right)$, cut plants and foliar spray with IAA at 40 days after cutting $\left(\mathrm{T}_{3}\right)$ and foliar spray with IAA twice at 20 and 40 days $\left(\mathrm{T}_{4}\right)$. The used concentration of IAA was 400 $\mathrm{ppm}$, the volume of water was one liter / plot, $0.5 \%$ wetting agent of tween 20 was used. The recommendations of other agronomic practices for barley production were applied. Data recorded were:-

\section{I. Growth characteristics}

Plant height $(\mathrm{cm})$, spike length $(\mathrm{cm})$ and Flag leaf area $\left(\mathrm{cm}^{2}\right)$ at 90 days after sowing (DAS) wear determined. Leaf area $\left(\mathrm{cm}^{2}\right)$ at 90 days after sowing (DAS) was calculated according to Strickler (1964). Total chlorophyll content of the leaves at 75 DAS was determined according to Witham et al. (1971).

\subsection{Yield and its component characteristics}

At harvest, the number of spikes $\mathrm{m}^{-2}$, number of kernels per spike, spike kernels weight (g), 
1000-kernel weight $(\mathrm{g})$, straw yield $\left(\mathrm{t} \mathrm{fed}^{-1}\right)$, grain yield $\left(\right.$ ard $\left.\mathrm{fed}^{-1}\right)$ and mean green and dry forage yield $\left(\mathrm{t} \mathrm{fed}^{-1}\right)$ were determined.

\subsection{Chemical components}

Crude protein and carbohydrates\% in grains
(Snedecor and Cochran, 1989)and found not significantly different. Then, the discussion of the obtained results was carried out on the basis of combined analysis values.

Table (1): Physical and chemical analyses of the experimental site.

\begin{tabular}{|l|c|c|}
\hline \multicolumn{1}{|c|}{ Trial } & $\mathbf{2}$ & $\mathbf{2 0 1 4 / 2 0 1 5}$ \\
\hline Soil type & Sandy & Sandy \\
Coarse sand & 83.15 & 82.86 \\
Fine sand & 10.35 & 10.64 \\
Silt\% & 1.40 & 1.50 \\
Clay\% & 5.10 & 5.00 \\
Organic matter & 0.66 & 0.63 \\
CaCo & 0.46 & 0.48 \\
PH & 7.42 & 7.70 \\
EC (dsm-1) & 0.10 & 0,13 \\
\hline \multicolumn{2}{|c|}{ Chemical analysis } \\
\hline Available N (ppm) & 27.49 & 35.20 \\
Available P (ppm) & 6.20 & 8.30 \\
Available K (ppm) & 70.50 & 83.00 \\
Available Fe (ppm) & 1.98 & 2.07 \\
Available Zn (ppm) & 0.7 & 0.8 \\
Available Mn (ppm) & 1.63 & 1.78 \\
\hline *Textural classes according to the triangular diagram.
\end{tabular}

C.F. Soil and Water Research Institute, A. R. C. Egypt.

were evaluated using the standard methods of A. O. A. C. (2000).

\section{4. Economic evaluation}

Partial budgeting was used to calculate the costs that vary (LE), gross benefit (LE) pound, net benefit (LE) and difference in net benefit (LE) of the four treatments $\left(\mathrm{T}_{1}, \mathrm{~T}_{2}, \mathrm{~T}_{3}\right.$ and $\left.\mathrm{T}_{4}\right)$. The marginal rate of return (MRR\%) was calculated as marginal net benefit (i.e., the differences in net benefit between grain yield and green forage yield / differences in costs that vary between grain yield and green forage yield) X 100 (CIMMYT, 1988).

Data of the two seasons were combined and statistically analyzed according to Steel and Torrie (1980) using MSTAT-C program computer program var. 4 (1986). Means were compared using the least significant difference (LSD) test at 0.05 level probabilities. The comparison of error mean squares between the two seasons for all traits was done with the help of Bartlett's test of homogeneity of variances

\section{RESULTS AND DISCUSSION}

\section{I. Growth characteristics}

Results in Tables (2 and 3) show that spike length and total chlorophyll were significantly affected by cultivars, treatments and their interactions in both seasons and combined analysis of the two seasons. Plant height $(\mathrm{cm})$ and leaf area $\left(\mathrm{cm}^{2}\right)$ at 90 DAS were significantly affected by treatments in both seasons and combined analysis of the two seasons.

Data in Tables (2 and 3) show that Giza 2000 had the highest values of spike length, total chlorophyll and flag area $\left(\mathrm{cm}^{2}\right)$ at 90 (DAS). Meanwhile, cultivar Giza 134 had the highest values of plant height (Table 2).

The control treatment (no cutting and without spray IAA) surpassed the other treatments in all growth traits under study. In comparison with the control treatment and the second treatment (cutting plants without IAA spraying), values of all the traits under study significantly decreased by cutting plants. Similar trend was 
recorded by Royo and Tribo (1997), Al-Satari et al. (2001) and Sharma (1998). Concerning the effect of foliar spray of barley with indole acetic acid (IAA) after cutting plants either once at 20 days $\left(\mathrm{T}_{2}\right)$, and at 40 days $\left(\mathrm{T}_{3}\right)$ or twice at 20 and 40 days $\left(\mathrm{T}_{4}\right)$ significantly increased in all growth characteristics under study compared with the first treatment $T_{1}$ (cutting plants without foliar spray of IAA). Significant effects were recorded between spraying barley plants with IAA twice and spraying barley plants with IAA once 20 days after cutting in all growth characteristics. While, plants treated with IAA twice had significant increases in all growth characteristics compared to the plants sprayed with IAA at 40 days after cutting. Plants treated with IAA at 20 days significantly surpassed the other treated at 40 days after cutting in all growth characteristics under study while, the differences between the two treatments in all growth traits under study were insignificant affected. The data obtained in this study concerning growth parameters are in good agreement with these obtained by Baz et al. (1984), Velu (1999), Govindan and Thirumurugan (2000) and Abdo and AbdelAziz, (2009) who stated that plants treated with foliar spray 50,100 or 150 ppm IAA showed pronounced increase in their vegetative growth, flag area and dry matter. They added that plants sprayed with NAA at $40 \mathrm{ppm}$ had significant increases in growth characters. Moreover, they stated that $150+60 \mathrm{ppm}(\mathrm{IAA}+\mathrm{NAA})$ gave the highest values of the growth parameters. While, the results of photosynthetic pigments were consistent with those obtained by Kalarani and Jeyakumar, (1998) who used NAA, and Senthil et al. (2003) who used 100 ppm IAA or 40 ppm NAA.

Table (2): Effect of foliar spray with IAA on plant height $(\mathrm{cm})$ and spike length $(\mathrm{cm})$ of three barley cultivars in both seasons and combined over two seasons.

\begin{tabular}{|c|c|c|c|c|c|c|c|}
\hline \multirow{2}{*}{$\begin{array}{l}\text { Cultivar } \\
\text { (C) }\end{array}$} & \multirow{2}{*}{$\begin{array}{l}\text { Treatment } \\
\text { (T) }\end{array}$} & \multicolumn{3}{|c|}{ Plant height $(\mathrm{cm})$} & \multicolumn{3}{|c|}{ Spike length $(\mathrm{cm})$} \\
\hline & & $2013 / 14$ & $2014 / 15$ & Comb. & $2013 / 14$ & $2014 / 15$ & Comb. \\
\hline \multirow{5}{*}{$\begin{array}{l}\text { Giza } 133 \\
\quad\left(\mathbf{C}_{1}\right)\end{array}$} & $\mathrm{T}_{0}$ & 70.7 & 72.33 & 71.52 & 6.79 & 6.71 & 6.75 \\
\hline & $\mathrm{T}_{1}$ & 63.33 & 65.55 & 64.44 & 5.33 & 5.66 & 5.50 \\
\hline & $\frac{1}{T_{2}}$ & 66.75 & 68.63 & 67.69 & 5.93 & 6.25 & 6.09 \\
\hline & $\mathrm{T}_{3}$ & 65.67 & 62.54 & 64.11 & 5.53 & 5.73 & 5.63 \\
\hline & $\mathrm{T}_{4}$ & 68.67 & 69.33 & 69.00 & 6.55 & 6.35 & 6.45 \\
\hline \multicolumn{2}{|c|}{ Mean of cultivar $\left(C_{1}\right)$} & 67.02 & 67.68 & 67.35 & 6.03 & 6.14 & 6.08 \\
\hline \multirow{5}{*}{$\begin{array}{c}\text { Giza } 134 \\
\left(C_{2}\right)\end{array}$} & $\mathrm{T}_{0}$ & 79.67 & 78.33 & 79.00 & 7.67 & 7.79 & 7.73 \\
\hline & $\mathrm{T}_{1}$ & 71 & 68.33 & 69.67 & 6.33 & 6.3 & 6.32 \\
\hline & $\mathrm{T}_{2}$ & 75.33 & 76.33 & 75.83 & 6.85 & 6.8 & 6.83 \\
\hline & $\mathrm{T}_{3}$ & 68.67 & 74.67 & 71.67 & 6.53 & 6.67 & 6.60 \\
\hline & $\mathrm{T}_{4}$ & 77.6 & 77.67 & 77.64 & 6.96 & 7.15 & 7.06 \\
\hline \multicolumn{2}{|c|}{ Mean of cultivar $\left(C_{2}\right)$} & 74.45 & 75.07 & 74.76 & 6.87 & 6.94 & 6.91 \\
\hline \multirow{5}{*}{$\begin{array}{c}\text { Giza } 2000 \\
\left(C_{3}\right)\end{array}$} & $\mathrm{T}_{0}$ & 72.33 & 75.22 & 73.78 & 7.93 & 7.89 & 7.91 \\
\hline & $\mathrm{T}_{1}$ & 64.33 & 66.3 & 65.32 & 6.53 & 6.52 & 6.53 \\
\hline & $\mathrm{T}_{2}$ & 67.55 & 70.45 & 69.00 & 6.92 & 6.89 & 6.91 \\
\hline & $\mathrm{T}_{3}$ & 66.37 & 69.54 & 67.96 & 6.85 & 6.88 & 6.87 \\
\hline & $\mathrm{T}_{4}$ & 70.67 & 72.33 & 71.50 & 7.1 & 7.18 & 7.14 \\
\hline \multicolumn{2}{|c|}{ Mean of cultivar $\left(\mathrm{C}_{3}\right)$} & 68.25 & 70.77 & 69.51 & 7.07 & 7.07 & 7.07 \\
\hline \multirow{5}{*}{$\begin{array}{c}\text { Mean of } \\
\text { treatments } \\
\text { (T) }\end{array}$} & $\mathrm{T}_{0}$ & 74.23 & 75.29 & 74.76 & 7.46 & 7.46 & 7.46 \\
\hline & $\mathrm{T}_{1}$ & 66.22 & 66.73 & 66.48 & 6.06 & 6.16 & 6.11 \\
\hline & $\mathrm{T}_{2}$ & 69.88 & 71.80 & 70.84 & 6.57 & 6.65 & 6.61 \\
\hline & $\mathrm{T}_{3}$ & 66.90 & 68.92 & 67.91 & 6.30 & 6.43 & 6.37 \\
\hline & $\mathrm{T}_{4}$ & 72.31 & 73.11 & 72.71 & 6.87 & 6.89 & 6.88 \\
\hline \multicolumn{2}{|c|}{ L. S. D at 0.05 for $(C)$} & N.S & N.S & N. S & 0.73 & 0.75 & 0.51 \\
\hline \multicolumn{2}{|c|}{ L. S. D at 0.05 for (T) } & 7.38 & 7.81 & 5.29 & 0.51 & 0.55 & 0.37 \\
\hline \multicolumn{2}{|c|}{ L. S. D at 0.05 for $C \times T$} & N. S & N. S & N. S & 1.08 & 1.14 & 0.77 \\
\hline \multicolumn{2}{|l|}{ C. V\% } & 5.03 & 5.69 & 5.47 & 4.60 & 4.93 & 4.47 \\
\hline
\end{tabular}

$\mathrm{T}_{0}=$ No cut, $\mathrm{T}_{1}=$ cut plants without spray IAA, $\mathrm{T}_{2}=$ cut plants and foliar spray of IAA at 20 days after cut, $\mathrm{T}_{3}=$ cut plants and foliar spray of IAA at 40 days and $\mathrm{T}_{4}=$ cut plants and foliar spray of IAA twice at 20 and 40 days after cutting plant. 
Table (3): Effect of foliar spray with IAA on mean leaf area $\left(\mathrm{m}^{2}\right)$ at 90 days and total chlorophyll of three barley cultivars in both seasons and combined over two seasons.

\begin{tabular}{|c|c|c|c|c|c|c|c|}
\hline \multirow[t]{2}{*}{$\begin{array}{c}\text { Cultivar } \\
\text { ( C ) }\end{array}$} & \multirow[t]{2}{*}{$\begin{array}{l}\text { Treatment } \\
\text { ( T ) }\end{array}$} & \multicolumn{3}{|c|}{ Mean leaf area $\left(\mathrm{cm}^{2}\right)$ at 90 days } & \multicolumn{3}{|c|}{$\begin{array}{c}\text { Total chlorophyll of leaves at } 75 \\
\text { DAS }\left(\mathrm{mg} \mathrm{g}^{-1}\right)\end{array}$} \\
\hline & & $2013 / 2014$ & $2014 / 2015$ & Comb. & $2013 / 2014$ & $2014 / 2015$ & Comb. \\
\hline \multirow{5}{*}{$\begin{array}{l}\text { Giza } 133 \\
\quad(\mathrm{C} 1)\end{array}$} & $\mathrm{T}_{0}$ & 55.01 & 55.26 & 55.14 & 2.55 & 2.49 & 2.52 \\
\hline & $\mathrm{T}_{1}$ & 42.46 & 42.16 & 42.31 & 1.37 & 1.46 & 1.42 \\
\hline & $\mathrm{T}_{2}$ & 50.22 & 50.57 & 50.40 & 2.18 & 2.17 & 2.18 \\
\hline & $\mathrm{T}_{3}$ & 48.94 & 49.86 & 49.40 & 1.97 & 1.99 & 1.98 \\
\hline & $\mathrm{T}_{4}$ & 52.38 & 52.58 & 52.48 & 2.32 & 2.32 & 2.32 \\
\hline \multicolumn{2}{|l|}{ Mean } & 49.80 & 50.09 & 49.95 & 2.08 & 2.08 & 2.08 \\
\hline \multirow{5}{*}{$\begin{array}{l}\text { Giza } 134 \\
\quad(\mathrm{C} 2)\end{array}$} & $\mathrm{T}_{0}$ & 58.14 & 57.90 & 58.02 & 2.68 & 2.72 & 2.70 \\
\hline & $\mathrm{T}_{1}$ & 45.70 & 45.51 & 45.61 & 1.80 & 1.79 & 1.80 \\
\hline & $\mathrm{T}_{2}$ & 53.28 & 53.26 & 53.27 & 2.35 & 2.41 & 2.38 \\
\hline & $\mathrm{T}_{3}$ & 52.43 & 52.58 & 52.51 & 2.24 & 2.29 & 2.27 \\
\hline & $\mathrm{T}_{4}$ & 56.37 & 56.21 & 56.29 & 2.23 & 2.52 & 2.38 \\
\hline \multicolumn{2}{|l|}{ Mean } & 53.18 & 53.09 & 53.14 & 2.26 & 2.34 & 2.30 \\
\hline \multirow{5}{*}{$\begin{array}{l}\text { Giza } 2000 \\
\text { (C3) }\end{array}$} & $\mathrm{T}_{0}$ & 59.78 & 59.62 & 59.70 & 2.86 & 2.87 & 2.87 \\
\hline & $\mathrm{T}_{1}$ & 48.12 & 45.75 & 46.94 & 1.90 & 1.94 & 1.92 \\
\hline & $\mathrm{T}_{2}$ & 55.23 & 55.39 & 55.31 & 2.35 & 2.52 & 2.50 \\
\hline & $T_{3}$ & 53.92 & 54.55 & 54.24 & 2.19 & 2.33 & 2.44 \\
\hline & $\mathrm{T}_{4}$ & 57.24 & 57.44 & 57.34 & 2.55 & 2.70 & 2.63 \\
\hline \multicolumn{2}{|l|}{ Mean } & 54.86 & 54.55 & 54.71 & 2.37 & 2.47 & 2.63 \\
\hline \multirow{5}{*}{$\begin{array}{l}\text { Mean of } \\
\text { treatments } \\
\text { (T) }\end{array}$} & $\mathrm{T}_{0}$ & 57.64 & 57.59 & 57.62 & 2.70 & 2.69 & 2.70 \\
\hline & $\mathrm{T}_{1}$ & 45.43 & 44.47 & 44.95 & 1.69 & 1.72 & 1.70 \\
\hline & $\mathrm{T}_{2}$ & 52.91 & 53.07 & 52.99 & 2.27 & 2.37 & 2.32 \\
\hline & $\mathrm{T}_{3}$ & 51.76 & 52.33 & 52.05 & 2.13 & 2.20 & 2.17 \\
\hline & $\mathrm{T}_{4}$ & 55.33 & 55.41 & 55.37 & 2.36 & 2.48 & 2.42 \\
\hline \multicolumn{2}{|c|}{ L. S. D at 0.05 for C } & N. S & N. S & N. S & 0.14 & 0.18 & 0.11 \\
\hline \multicolumn{2}{|c|}{ L. S. D at 0.05 for $\mathrm{T}$} & 5.81 & 5.55 & 3.99 & 0.12 & 0.15 & 0.09 \\
\hline \multirow{2}{*}{\multicolumn{2}{|c|}{$\begin{array}{l}\text { L. S. D at } 0.05 \mathrm{C} \times \mathrm{T} \\
\text { C. V\% }\end{array}$}} & N. S & N. S & N. S & 0.20 & 0.25 & 1.6 \\
\hline & & 3.94 & 3.69 & 3.83 & 4.74 & 4.37 & 4.47 \\
\hline
\end{tabular}

$\mathrm{T}_{0}=$ No cut, $\mathrm{T}_{1}=$ cut plants without spray IAA, $\mathrm{T}_{2}=$ cut plants and foliar spray of IAA at 20 days after cut, $\mathrm{T}_{3}=$ cut plants and foliar spray of IAA at 40 days and $\mathrm{T}_{4}=$ cut plants and foliar spray of IAA twice at 20 and 40 days after cutting plant..

Moreover, the best interaction between cultivar $\mathrm{x}$ treatment was $c v$. Giza $2000 \mathrm{x}$ cut plants and foliar spray of IAA twice at 20 and 40 days after cutting $\left(\mathrm{T}_{4}\right)$ Tables (2 and 3 ) in all growth characteristics under study. Except for, plant height the best interaction between cultivar $\mathrm{x}$ treatment was $c v$. Giza $134 \mathrm{x}$ cut plants and foliar spray of IAA twice at 20 and 40 days after cutting $\left(\mathrm{T}_{4}\right)$ Table (2).

\section{2. Yield and its components}

Results in Tables (4, 5 and 6) show for number of spikes $/ \mathrm{m}^{2}$, number of kernels/spike, spike kernels weight (g), 1000-kernel weight (g) and grain yield (ard fed 1 ) were significantly affected by cultivars, treatments and their interactions in both seasons and combined analysis. Straw yield $\left(\mathrm{t} \mathrm{fed}^{-1}\right)$ was significantly affected by treatments and their interactions in both seasons and combined analysis.

The data in Tables (4,5 and 6) show that Giza 2000 cultivar had the highest values for number of spikes $/ \mathrm{m}^{2}$, the number of kernels/spike, spike kernels weight (g), 1000kernel weight $(\mathrm{g})$, straw yield $\left(\mathrm{t} \mathrm{fed}^{-1}\right)$ and grain yield $\left(\operatorname{ard} \mathrm{fed}^{-1}\right)$.

The control treatment $\left(\mathrm{T}_{0}\right)$ significantly surpassed the other treatments $\left(\mathrm{T}_{1}, \mathrm{~T}_{2}, \mathrm{~T}_{3}\right.$ and $\left.\mathrm{T}_{4}\right)$ in all characteristics. These results are in agreement with those obtained by Royo (1999), Yau (1999), Al-Satari et al. (2001) and Sharma (2002). Foliar spray of barley with IAA 20 and 40 days after cutting gave significant increases for all traits compared to cutting plants without foliar spray of IAA $\left(\mathrm{T}_{1}\right)$. In comparison between spraying IAA at 20 and 40 days after cutting treatments, early spray of IAA caused significantly increases in number of spikes $/ \mathrm{m}^{2}$, number of kernels/spike, spike kernels weight $(\mathrm{g})$, 1000- kernel weight $(\mathrm{g})$, straw yield $\left(\mathrm{t} \mathrm{fed}^{-1}\right)$ and grain yield (ard fed 1$)$. Spraying plants with IAA twice had significant increases in all characteristics under study. While, 
Table (4): Effect of foliar spray with IAA on the number of spikes $\mathrm{m}^{2}$ and the number of kernels spike- ${ }^{1}$ of three barley cultivars in both seasons and combined over two seasons.

\begin{tabular}{|c|c|c|c|c|c|c|c|}
\hline \multirow{2}{*}{$\begin{array}{c}\text { Cultivar } \\
\text { (C) }\end{array}$} & \multirow{2}{*}{$\begin{array}{l}\text { Treatment } \\
\text { (T) }\end{array}$} & \multicolumn{3}{|c|}{ Number of spikes $\mathrm{m}^{2}{ }^{2}$} & \multicolumn{3}{|c|}{ Number of kernels spike- ${ }^{1}$} \\
\hline & & $2013 / 2014$ & $2014 / 2015$ & Comb. & $2013 / 2014$ & $2014 / 2015$ & Comb. \\
\hline \multirow{5}{*}{$\begin{array}{c}\text { Giza } \\
133\left(C_{1}\right)\end{array}$} & $\mathrm{T}_{0}$ & 335.2 & 343.25 & 339.23 & 49.40 & 48.35 & 48.88 \\
\hline & $\mathrm{T}_{1}$ & 210.15 & 225.21 & 217.68 & 35.00 & 37.45 & 36.23 \\
\hline & $\mathrm{T}_{2}$ & 270.30 & 283.11 & 276.71 & 38.50 & 39.60 & 39.05 \\
\hline & $T_{3}$ & 264.41 & 255.43 & 259.92 & 33.70 & 35.41 & 34.56 \\
\hline & $\mathrm{T}_{4}$ & 305.73 & 315.23 & 310.48 & 44.83 & 46.91 & 45.87 \\
\hline \multicolumn{2}{|c|}{ Mean } & 277.16 & 284.45 & 280.80 & 40.29 & 41.54 & 40.92 \\
\hline \multirow{5}{*}{$\begin{array}{c}\text { Giza } 134 \\
\left(\mathbf{C}_{2}\right)\end{array}$} & $\mathrm{T}_{0}$ & 363.22 & 359.36 & 361.29 & 53.41 & 55.22 & 54.32 \\
\hline & $\mathrm{T}_{1}$ & 250.41 & 233.53 & 241.97 & 38.67 & 39.41 & 39.04 \\
\hline & $\mathrm{T}_{2}$ & 293.11 & 309.25 & 301.18 & 44.65 & 45.31 & 44.98 \\
\hline & $\mathrm{T}_{3}$ & 271.51 & 275.43 & 273.47 & 41.43 & 43.63 & 42.53 \\
\hline & $\mathrm{T}_{4}$ & 327.22 & 335.41 & 331.32 & 48.30 & 50.41 & 49.36 \\
\hline \multicolumn{2}{|c|}{ Mean } & 301.09 & 302.60 & 301.85 & 45.29 & 46.80 & 46.05 \\
\hline \multirow{5}{*}{$\begin{array}{c}\text { Giza } 2000 \\
\left(\mathbf{C}_{3}\right)\end{array}$} & $\mathrm{T}_{0}$ & 439.55 & 393.41 & 416.48 & 56.30 & 58.20 & 57.25 \\
\hline & $\mathrm{T}_{1}$ & 311.25 & 322.11 & 316.68 & 40.22 & 43.71 & 41.97 \\
\hline & $\mathrm{T}_{2}$ & 365.41 & 379.20 & 372.31 & 49.61 & 48.49 & 49.05 \\
\hline & $\mathrm{T}_{3}$ & 350.30 & 349.60 & 349.95 & 46.33 & 45.60 & 45.97 \\
\hline & $\mathrm{T}_{4}$ & 401.60 & 412.81 & 407.20 & 52.35 & 55.41 & 53.88 \\
\hline \multicolumn{2}{|c|}{ Mean } & 373.62 & 371.43 & 372.53 & 48.96 & 50.28 & 49.62 \\
\hline \multirow{5}{*}{$\begin{array}{c}\text { Mean of } \\
\text { treatments } \\
\text { (T) }\end{array}$} & $\mathrm{T}_{0}$ & 379.32 & 365.34 & 372.33 & 53.04 & 53.92 & 53.48 \\
\hline & $\mathrm{T}_{1}$ & 257.27 & 260.28 & 258.78 & 37.96 & 40.19 & 39.08 \\
\hline & $\mathrm{T}_{2}$ & 309.61 & 323.85 & 316.73 & 44.25 & 44.47 & 44.36 \\
\hline & $\mathrm{T}_{3}$ & 295.41 & 293.49 & 294.45 & 40.49 & 41.55 & 41.02 \\
\hline & $\mathrm{T}_{4}$ & 344.85 & 354.48 & 349.67 & 48.49 & 50.91 & 49.70 \\
\hline \multicolumn{2}{|c|}{ L. S. D at 0.05 for (C) } & 18.65 & 16.48 & 12.19 & 4.92 & 5.07 & 3.49 \\
\hline \multirow{2}{*}{\multicolumn{2}{|c|}{ L. S. D at 0.05 for $(\mathrm{T})$}} & 10.08 & 9.76 & 7.01 & 3.43 & 3.52 & 2.43 \\
\hline & & 27.45 & 26.90 & 19.15 & 7.27 & 7.49 & 5.16 \\
\hline \multicolumn{2}{|c|}{$\begin{array}{l}\text { L. S. D at } 0.05 \text { for } C \times \text { T } \\
\text { C. V\% }\end{array}$} & 11.69 & 11.60 & 11.64 & 4.73 & 5.31 & 5.05 \\
\hline
\end{tabular}

$\mathrm{T}_{0}=$ No cut, $\mathrm{T}_{1}=$ cut plants without spray IAA, $\mathrm{T}_{2}=$ cut plants and foliar spray of IAA at 20 days after cut, $\mathrm{T}_{3}=$ cut plants and foliar spray of IAA at 40 days and $\mathrm{T}_{4}=$ cut plants and foliar spray of IAA twice at 20 and 40 days after cutting plant.

plants sprayed with IAA twice surpassed the others sprayed at 40 days after cutting in all characteristics. In comparison with the control, no cut and without spray IAA $\left(\mathrm{T}_{0}\right)$ and spraying plants with IAA twice after cutting $\left(\mathrm{T}_{4}\right)$, no significant differences were recorded in all the characteristics under study. It is clear that plants recovery after forage removal was affected by foliar application of IAA. In addition, cutting plants treatment spraying plants with IAA twice after cutting $\left(\mathrm{T}_{4}\right)$ produced an average of 6.00 ton fed ${ }^{-1}$ green forage or 2.20 ton fed ${ }^{-1}$ dry yield per fed (Table 7). In this connection, similar results were observed by Barsoum (1994); Senthil et al. (2003), Abdel-Aziz et al. (2004), Zaki and Radwan (2011), Tiwari et al. (2011) and Mona et al. (2013).

Moreover, the best interaction between cultivar $\mathrm{x}$ treatment was $c v$. Giza $2000 \mathrm{x}$ cut plants and foliar spray of IAA twice at 20 and 40 days after cutting $\left(\mathrm{T}_{4}\right)$ Tables $(4,5$ and 6$)$ in all yield characteristics under study.

\subsection{Chemical components}

Results of chemical components Table (8) i. e carbohydrates $\%$ and proteins $\%$ were highly significantly affected by cultivars, treatments and their interactions. Giza 2000 had the highest carbohydrates $\%$ and proteins $\%$ (Table 8 ). The control (no cut plants) surpassed the other treatments for carbohydrate $\%$ and protein $\%$. In addition, the highest treatment was cutting plants and foliar spray of IAA twice at 20 and 40 days after cutting $\left(\mathrm{T}_{4}\right)$ (Table 8). Moreover, the best interaction between cultivar $\mathrm{x}$ treatment was $c v$. Giza $2000 \mathrm{x}$ cut plants and foliar spray of IAA twice at 20 and 40 days after cutting $\left(\mathrm{T}_{4}\right)$ Table (8). Data showed that, for carbohydrate and protein $\%$ the first treatment (no-cutting and without foliar spray of barley with IAA) gave the highest values compared to to $T_{1}, T_{2}, T_{3}$ and $\mathrm{T}_{4}$ for all chemical characteristics under study (Table 8). Foliar chemical characteristics in both 
Table (5): Effect of foliar spray with IAA on spike kernel weight (g) and1000-kernel weight (g) of three barley cultivars in both seasons and combined over two seasons.

\begin{tabular}{|c|c|c|c|c|c|c|c|}
\hline \multirow[t]{2}{*}{ Cultivar (C) } & \multirow{2}{*}{$\begin{array}{l}\text { Treatment } \\
\text { (T) }\end{array}$} & \multicolumn{3}{|c|}{ Spike kernels weight (g) } & \multicolumn{3}{|c|}{ 1000-kernel weight (g) } \\
\hline & & $\begin{array}{c}2013 / \\
2014 \\
\end{array}$ & $\begin{array}{l}2014 / \\
2015 \\
\end{array}$ & Comb. & $\begin{array}{l}2013 / \\
2014 \\
\end{array}$ & $\begin{array}{l}2014 / \\
2015 \\
\end{array}$ & Comb. \\
\hline \multirow{5}{*}{ Giza $133\left(C_{1}\right)$} & $\mathrm{T}_{0}$ & 1.40 & 1.45 & 1.43 & 36.20 & 35.87 & 36.03 \\
\hline & $\mathrm{T}_{1}$ & 1.17 & 1.12 & 1.15 & 28.34 & 29.42 & 28.88 \\
\hline & $\mathrm{T}_{2}$ & 1.29 & 1.32 & 1.31 & 33.43 & 32.99 & 33.21 \\
\hline & $\mathrm{T}_{3}$ & 1.22 & 1.27 & 1.25 & 30.34 & 29.91 & 30.13 \\
\hline & $\mathrm{T}_{4}$ & 1.36 & 1.38 & 1.37 & 33.51 & 33.33 & 33.42 \\
\hline \multicolumn{2}{|c|}{ Mean } & 1.29 & 1.31 & 1.30 & 32.02 & 32.30 & 32.16 \\
\hline \multirow{5}{*}{ Giza $134\left(C_{2}\right)$} & $\mathrm{T}_{0}$ & 1.66 & 1.68 & 1.67 & 37.81 & 37.72 & 37.77 \\
\hline & $\mathrm{T}_{1}$ & 1.23 & 1.25 & 1.24 & 30.24 & 31.37 & 30.81 \\
\hline & $\mathrm{T}_{2}$ & 1.35 & 1.33 & 1.34 & 34.65 & 34.35 & 34.50 \\
\hline & $\mathrm{T}_{3}$ & 1.27 & 1.28 & 1.28 & 31.43 & 33.34 & 32.39 \\
\hline & $\mathrm{T}_{4}$ & 1.47 & 1.43 & 1.45 & 35.08 & 35.42 & 35.25 \\
\hline \multicolumn{2}{|c|}{ Mean } & 1.40 & 1.39 & 1.40 & 33.84 & 34.44 & 34.14 \\
\hline \multirow{5}{*}{ Giza $2000\left(C_{3}\right)$} & $\mathrm{T}_{0}$ & 1.70 & 1.72 & 1.71 & 38.52 & 37.81 & 38.17 \\
\hline & $\mathrm{T}_{1}$ & 1.26 & 1.27 & 1.27 & 32.62 & 32.41 & 32.52 \\
\hline & $\mathrm{T}_{2}$ & 1.42 & 1.43 & 1.43 & 35.20 & 35.71 & 35.46 \\
\hline & $\mathrm{T}_{3}$ & 1.38 & 1.39 & 1.39 & 34.79 & 34.90 & 34.85 \\
\hline & $\mathrm{T}_{4}$ & 1.65 & 1.67 & 1.66 & 36.33 & 36.89 & 36.61 \\
\hline \multicolumn{2}{|c|}{ Mean } & 1.48 & 1.50 & 1.49 & 35.49 & 35.54 & 35.52 \\
\hline \multirow{5}{*}{$\begin{array}{l}\text { Mean of } \\
\text { treatments } \\
\text { (T) }\end{array}$} & $\mathrm{T}_{0}$ & 1.59 & 1.62 & 1.60 & 37.51 & 37.13 & 37.32 \\
\hline & $\mathrm{T}_{1}$ & 1.22 & 1.21 & 1.22 & 30.40 & 31.07 & 30.74 \\
\hline & $\mathrm{T}_{2}$ & 1.35 & 1.36 & 1.36 & 34.43 & 34.33 & 34.38 \\
\hline & $\mathrm{T}_{3}$ & 1.29 & 1.31 & 1.31 & 32.19 & 32.72 & 32.46 \\
\hline & $\mathrm{T}_{4}$ & 1.49 & 1.49 & 1.49 & 34.97 & 35.21 & 35.09 \\
\hline \multicolumn{2}{|c|}{ L. S. D at 0.05 for $(C)$} & 0.15 & 0.16 & 0.11 & 2.56 & 2.49 & 1.76 \\
\hline \multicolumn{2}{|c|}{ L. S. D at 0.05 for (T) } & 0.11 & 0.12 & 0.08 & 1.78 & 1.73 & 1.22 \\
\hline \multicolumn{2}{|c|}{ L. S. D at 0.05 for $C \times T$} & 0.22 & 0.23 & 0.16 & 3.78 & 3.68 & 2.61 \\
\hline \multicolumn{2}{|l|}{ C. V\% } & 2.06 & 2.27 & 2.16 & 3.00 & 2.72 & 2.86 \\
\hline
\end{tabular}

$\mathrm{T}_{0}=$ No cut, $\mathrm{T}_{1}=$ cut plants without spray IAA, $\mathrm{T}_{2}=$ cut plants and foliar spray of IAA at 20 days after cut, $\mathrm{T}_{3}=$ cut plants and foliar spray of IAA at 40 days and $\mathrm{T}_{4}=$ cut plants and foliar spray of IAA twice at 20 and 40 days after cutting plant. 
Table( 6): Effect of foliar spray with IAA on straw yield (ton fed- ${ }^{\mathbf{1}}$ ) and grain yield (ard fed- ${ }^{\mathbf{1}}$ ) of three barley cultivars in both seasons and combined over two seasons.

\begin{tabular}{|c|c|c|c|c|c|c|c|}
\hline \multirow{2}{*}{$\begin{array}{c}\text { Cultivars } \\
\text { (C) }\end{array}$} & \multirow{2}{*}{$\begin{array}{l}\text { Treatments* } \\
\text { (T) }\end{array}$} & \multicolumn{3}{|c|}{ Straw yield (ton fed- ${ }^{1}$ ) } & \multicolumn{3}{|c|}{ Grain yield (ard fed- $^{1}$ ) } \\
\hline & & $2013 / 2014$ & $2014 / 2015$ & Comb. & $2013 / 2014$ & $2014 / 2015$ & Comb. \\
\hline \multirow{5}{*}{$\begin{array}{l}\text { Giza } \\
133\left(C_{1}\right)\end{array}$} & $\mathrm{T}_{0}$ & 2.50 & 2.65 & 2.58 & 8.53 & 8.43 & 8.48 \\
\hline & $\mathrm{T}_{1}$ & 1.53 & 1.43 & 1.48 & 5.20 & 5.35 & 5.28 \\
\hline & $\mathrm{T}_{2}$ & 2.13 & 2.19 & 2.16 & 7.33 & 7.55 & 7.44 \\
\hline & $\mathrm{T}_{3}$ & 2.05 & 2.10 & 2.08 & 7.05 & 7.16 & 7.11 \\
\hline & $\mathrm{T}_{4}$ & 2.37 & 2.25 & 2.31 & 7.80 & 7.75 & 7.78 \\
\hline \multicolumn{2}{|c|}{ Mean } & 2.12 & 2.12 & 2.12 & 7.18 & 7.25 & 7.22 \\
\hline \multirow{5}{*}{$\begin{array}{l}\text { Giza } \\
134\left(C_{2}\right)\end{array}$} & $\mathrm{T}_{0}$ & 2.75 & 2.83 & 2.79 & 8.85 & 8.69 & 8.77 \\
\hline & $\mathrm{T}_{1}$ & 1.65 & 1.79 & 1.72 & 5.66 & 6.07 & 5.87 \\
\hline & $\mathrm{T}_{2}$ & 2.26 & 2.22 & 2.24 & 7.63 & 7.77 & 7.70 \\
\hline & $\mathrm{T}_{3}$ & 2.16 & 2.11 & 2.14 & 7.23 & 7.49 & 7.36 \\
\hline & $\mathrm{T}_{4}$ & 2.46 & 2.38 & 2.42 & 8.11 & 8.23 & 8.17 \\
\hline \multicolumn{2}{|c|}{ Mean } & 2.26 & 2.27 & 2.26 & 7.50 & 7.65 & 7.57 \\
\hline \multirow{5}{*}{$\begin{array}{l}\text { Giza } \\
2000\left(C_{3}\right)\end{array}$} & $\mathrm{T}_{0}$ & 3.77 & 3.56 & 3.67 & 9.53 & 9.85 & 9.69 \\
\hline & $\mathrm{T}_{1}$ & 1.85 & 1.93 & 1.89 & 6.30 & 6.44 & 6.37 \\
\hline & $\mathrm{T}_{2}$ & 2.73 & 2.65 & 2.69 & 8.60 & 8.56 & 8.58 \\
\hline & $\mathrm{T}_{3}$ & 2.39 & 2.53 & 2.46 & 8.30 & 8.48 & 8.39 \\
\hline & $\mathrm{T}_{4}$ & 3.13 & 3.09 & 3.11 & 9.13 & 9.29 & 9.21 \\
\hline \multicolumn{2}{|c|}{ Mean } & 2.77 & 2.75 & 2.76 & 8.37 & 8.52 & 8.45 \\
\hline \multirow{5}{*}{$\begin{array}{l}\text { Mean of } \\
\text { treatments } \\
(\mathrm{T})\end{array}$} & $\mathrm{T}_{0}$ & 3.00 & 3.01 & 3.01 & 8.97 & 8.99 & 8.98 \\
\hline & $\mathrm{T}_{1}$ & 1.68 & 1.72 & 1.70 & 5.72 & 5.95 & 5.84 \\
\hline & $\mathrm{T}_{2}$ & 2.37 & 2.35 & 2.36 & 7.85 & 7.96 & 7.91 \\
\hline & $\mathrm{T}_{3}$ & 2.20 & 2.25 & 2.23 & 7.53 & 7.71 & 7.62 \\
\hline & $\mathrm{T}_{4}$ & 2.65 & 2.57 & 2.61 & 8.35 & 8.42 & 8.39 \\
\hline \multicolumn{2}{|c|}{ L. S. D at 0.05 for $C$} & N.S & N.S & N.S & 0.91 & 0.98 & 0.66 \\
\hline \multicolumn{2}{|c|}{ L. S. D at 0.05 for $T$} & 0.72 & 0.74 & 0.51 & 0.65 & 0.63 & 0.45 \\
\hline \multicolumn{2}{|c|}{ L. S. D at $0.05 \mathrm{C} \times \mathrm{T}$} & 1.53 & 1.57 & 1.08 & 1.35 & 1.31 & 0.93 \\
\hline \multicolumn{2}{|l|}{ C. V\% } & 10.49 & 10.38 & 10.87 & 10.66 & 10.76 & 10.71 \\
\hline
\end{tabular}

Table (7): Mean of foliar spray with IAA on green forage yield $\left(\mathrm{t} \mathrm{fed}^{-1}\right)$ and dry forage yield $\left(\mathrm{t}\right.$ fed $\left.{ }^{-1}\right)$ of three barley cultivars in both seasons combined over two seasons.

\begin{tabular}{|c|c|c|c|c|c|c|c|}
\hline \multirow[t]{2}{*}{ Cultivar (C) } & \multirow{2}{*}{$\begin{array}{l}\text { Treatment } \\
\text { (T) }\end{array}$} & \multicolumn{3}{|c|}{ Green forage yield $\left(\mathrm{t} \mathrm{fed}^{-1}\right)$} & \multicolumn{3}{|c|}{ Dry forage yield $\left(\mathrm{t} \mathrm{fed}^{-1}\right)$} \\
\hline & & $2013 / 14$ & $2014 / 15$ & Comb. & $2013 / 14$ & $2014 / 15$ & Comb. \\
\hline \multirow{5}{*}{ Giza $133\left(C_{1}\right)$} & $\mathrm{T}_{0}$ & - & - & - & - & - & - \\
\hline & $\mathrm{T}_{1}$ & 5.17 & 5.10 & 5.14 & 1.05 & 1.06 & 1.05 \\
\hline & $\mathrm{T}_{2}$ & 5.36 & 5.39 & 5.38 & 1.08 & 1.08 & 1.08 \\
\hline & $\mathrm{T}_{3}$ & 5.30 & 5.32 & 5.31 & 1.07 & 1.07 & 1.07 \\
\hline & $\mathrm{T}_{4}$ & 5.54 & 5.46 & 5.50 & 1.09 & 1.09 & 1.1 \\
\hline \multicolumn{2}{|c|}{ Mean } & 5.34 & 5.32 & 5.33 & 1.07 & 1.08 & 1.07 \\
\hline \multirow{5}{*}{$\begin{array}{c}\text { Giza } 134 \\
\left(C_{2}\right)\end{array}$} & $\mathrm{T}_{0}$ & - & - & - & - & - & - \\
\hline & $\mathrm{T}_{1}$ & 5.76 & 5.66 & 5.71 & 1.06 & 1.07 & 1.06 \\
\hline & $\mathrm{T}_{2}$ & 5.85 & 5.90 & 5.88 & 1.08 & 1.08 & 1.08 \\
\hline & $\mathrm{T}_{3}$ & 5.73 & 5.76 & 5.75 & 1.07 & 1.09 & 1.08 \\
\hline & $\mathrm{T}_{4}$ & 5.93 & 5.96 & 5.95 & 2.03 & 2.04 & 2.08 \\
\hline \multicolumn{2}{|c|}{ Mean } & 5.82 & 5.82 & 5.82 & 1.08 & 1.08 & 1.08 \\
\hline \multirow{5}{*}{$\begin{array}{c}\text { Giza } 2000 \\
\quad\left(\mathbf{C}_{3}\right)\end{array}$} & $\mathrm{T}_{0}$ & - & - & - & - & - & - \\
\hline & $\mathrm{T}_{1}$ & 5.89 & 5.85 & 5.87 & 1.08 & 1.01 & 1.09 \\
\hline & $T_{2}$ & 6.69 & 6.75 & 6.72 & 2.01 & 2.09 & 2.02 \\
\hline & $\mathrm{T}_{3}$ & 6.53 & 6.43 & 6.48 & 2.05 & 2.05 & 2.03 \\
\hline & $\mathrm{T}_{4}$ & 6.81 & 6.93 & 6.87 & 2.03 & 2.04 & 2.06 \\
\hline \multicolumn{2}{|c|}{ Mean } & 6.48 & 6.49 & 6.49 & 2.09 & 2.07 & 2.02 \\
\hline \multirow{5}{*}{$\begin{array}{c}\text { Mean of } \\
\text { treatments } \\
(\mathrm{T})\end{array}$} & $\mathrm{T}_{0}$ & - & - & - & - & - & - \\
\hline & $\mathrm{T}_{1}$ & 5.61 & 5.54 & 5.57 & 1.08 & 1.04 & 1.07 \\
\hline & $\mathrm{T}_{2}$ & 5.97 & 6.01 & 5.99 & 1.09 & 1.08 & 1.09 \\
\hline & $\mathrm{T}_{3}$ & 5.85 & 5.84 & 5.85 & 1.01 & 1.06 & 1.09 \\
\hline & $\mathrm{T}_{4}$ & 6.09 & 6.12 & 6.11 & 2.06 & 2.05 & 2.02 \\
\hline
\end{tabular}

$\mathrm{T}_{0}=$ No cut, $\mathrm{T}_{1}=$ cut plants without spray IAA, $\mathrm{T}_{2}=$ cut plants and foliar spray of IAA at 20 days after cut, $\mathrm{T}_{3}=$ cut plants and foliar spray of IAA at 40 days and $\mathrm{T}_{4}=$ cut plants and foliar spray of IAA twice at 20 and 40 days after cut plant. 
seasons and combined analysis. While, plants sprayed with IAA twice $\left(\mathrm{T}_{4}\right)$ had significant increases in all chemical characteristics and IAA compared to plants sprayed with IAA once at 40 days $\left(\mathrm{T}_{3}\right)$ after cutting (Table 8). Similar results were recorded by Abdel-Aziz et al. (2004) and Mona et al. (2013). an increase in the difference in benefits between and marginal rate of return percentage (MRR \%) of grain and green forage yield of all cultivars in this study. Giza 2000 had the highest net benefit and marginal rate return percentage (MRR\%) (Table 9). Also, the highest net benefit and marginal rate return percentage (MRR \%) at

Table (8): Effect of foliar spray with IAA on plant carbohydrate and protein \% of three barley cultivars in both seasons and combined over two seasons.

\begin{tabular}{|c|c|c|c|c|c|c|c|}
\hline \multirow{2}{*}{$\begin{array}{c}\text { Cultivars } \\
\text { (C) }\end{array}$} & \multirow{2}{*}{$\begin{array}{l}\text { Treatments* } \\
\text { (T) }\end{array}$} & \multicolumn{3}{|c|}{ Carbohydrate \% } & \multicolumn{3}{|c|}{ Protein \% } \\
\hline & & 2013/14 & $2014 / 15$ & Comb. & 2013/14 & $2014 / 15$ & Comb. \\
\hline \multirow{5}{*}{$\begin{array}{c}\text { Giza } \\
133\left(C_{1}\right)\end{array}$} & $\mathrm{T}_{0}$ & 71.76 & 72.08 & 71.92 & 10.66 & 10.62 & 10.64 \\
\hline & $\mathrm{T}_{1}$ & 60.58 & 60.74 & 60.66 & 9.18 & 9.15 & 9.17 \\
\hline & $\mathrm{T}_{2}$ & 64.61 & 64.38 & 64.50 & 10.23 & 10.37 & 10.30 \\
\hline & $\mathrm{T}_{3}$ & 63.41 & 63.12 & 63.27 & 10.15 & 10.06 & 10.11 \\
\hline & $\mathrm{T}_{4}$ & 67.28 & 67.51 & 67.40 & 10.40 & 10.44 & 10.42 \\
\hline \multicolumn{2}{|c|}{ Mean } & 65.53 & 65.57 & 65.55 & 10.12 & 10.13 & 10.12 \\
\hline \multirow{5}{*}{$\begin{array}{c}\text { Giza } 134 \\
\quad\left(\mathbf{C}_{2}\right)\end{array}$} & $\mathrm{T}_{0}$ & 73.28 & 73.31 & 73.30 & 10.76 & 10.73 & 10.75 \\
\hline & $\mathrm{T}_{1}$ & 60.97 & 60.70 & 60.84 & 9.56 & 9.63 & 9.60 \\
\hline & $\mathrm{T}_{2}$ & 66.57 & 66.89 & 66.73 & 10.44 & 10.42 & 10.43 \\
\hline & $\mathrm{T}_{3}$ & 64.52 & 64.24 & 64.38 & 10.20 & 10.22 & 10.21 \\
\hline & $\mathrm{T}_{4}$ & 70.55 & 70.35 & 70.45 & 10.50 & 10.52 & 10.51 \\
\hline \multicolumn{2}{|c|}{ Mean } & 67.18 & 67.10 & 67.14 & 10.29 & 10.30 & 10.30 \\
\hline \multirow{5}{*}{$\begin{array}{c}\text { Giza } 2000 \\
\left(\mathbf{C}_{3}\right)\end{array}$} & $\mathrm{T}_{0}$ & 77.94 & 78.91 & 78.43 & 10.86 & 10.88 & 10.87 \\
\hline & $\mathrm{T}_{1}$ & 63.48 & 63.41 & 63.44 & 9.63 & 9.67 & 9.65 \\
\hline & $\mathrm{T}_{2}$ & 69.81 & 70.81 & 70.31 & 10.60 & 10.62 & 10.61 \\
\hline & $\mathrm{T}_{3}$ & 68.81 & 68.61 & 68.70 & 10.22 & 10.24 & 10.23 \\
\hline & $\mathrm{T}_{4}$ & 75.57 & 76.50 & 76.04 & 10.62 & 10.66 & 10.64 \\
\hline \multicolumn{2}{|c|}{ Mean } & 71.12 & 71.65 & 71.39 & 10.39 & 10.41 & 10.40 \\
\hline \multirow{5}{*}{$\begin{array}{l}\text { Mean of } \\
\text { treatments } \\
\text { (T) }\end{array}$} & $\mathrm{T}_{0}$ & 74.33 & 74.77 & 74.55 & 10.76 & 10.74 & 10.75 \\
\hline & $\mathrm{T}_{1}$ & 61.68 & 61.62 & 61.65 & 9.46 & 9.48 & 9.48 \\
\hline & $\mathrm{T}_{2}$ & 67.00 & 67.36 & 67.18 & 10.42 & 10.47 & 10.45 \\
\hline & $\mathrm{T}_{3}$ & 65.58 & 66.21 & 65.90 & 10.19 & 10.17 & 10.18 \\
\hline & $\mathrm{T}_{4}$ & 71.13 & 71.45 & 71.29 & 10.51 & 10.54 & 10.53 \\
\hline \multicolumn{2}{|c|}{ L. S. D at 0.05 for $(C)$} & 3.63 & 3.57 & 2.46 & N. S & N.S & N.S \\
\hline \multicolumn{2}{|c|}{ L. S. D at 0.05 for $(T)$} & 2.89 & 2.41 & 1.79 & 0.49 & 0.41 & 0.30 \\
\hline \multicolumn{2}{|c|}{ L. S. D at 0.05 for $C \times T$} & 4.54 & 4.70 & 3.15 & 0.75 & 0.79 & 0.52 \\
\hline \multicolumn{2}{|l|}{ C. V\% } & 4.41 & 4.64 & 4.49 & 3.93 & 4.15 & 4.04 \\
\hline
\end{tabular}

$* \mathrm{~T}_{0}=$ No cut, $\mathrm{T}_{1}=$ cut plants without spray IAA, $\mathrm{T}_{2}=$ cut plants and foliar spray of IAA at 20 days after cut, $\mathrm{T}_{3}=$ cut plants and foliar spray of IAA at 40 days and $\mathrm{T}_{4}=$ cut plants and foliar spray of IAA twice at 20 and 40 days after cut plant.

\section{4. Economic evaluation}

A nalysis of variance showed that there were significant differences of interaction between cultivars $\mathrm{x}$ treatments in combined analysis (Table 9). Data in Table 9 showed that there was treatment was cut plants and foliar spray of IAA twice at 20 and 40 days after cut using $\left(\mathrm{T}_{4}\right)$ in combined analysis 4069.6 LE and $369.51 \%$, respectively. 
Table (9): Effect of the partial budget of foliar spray with IAA of three barley cultivars combined over two seasons.

\begin{tabular}{|c|c|c|c|c|c|c|}
\hline \multirow{2}{*}{$\begin{array}{c}\text { Cultivar } \\
\text { (C) }\end{array}$} & \multirow[t]{2}{*}{ Parameters } & \multicolumn{5}{|c|}{ Treatments $(\mathbf{T})^{*}$} \\
\hline & & $\mathbf{T}_{\mathbf{0}}$ & $\mathbf{T}_{1}$ & $\mathbf{T}_{2}$ & $\mathbf{T}_{3}$ & $\mathbf{T}_{4}$ \\
\hline & Mean of grain yield $\left(\operatorname{ard} /\right.$ fed $\left.^{-1}\right)$ & 8.48 & 5.28 & 7.44 & 7.11 & 7.78 \\
\hline Giza & Gross benefit (LE) & 3052.8 & 1897.2 & 2678.4 & 2545.2 & 2779.2 \\
\hline 133 & Costs that vary (LE) & 1000 & 1000 & 1050 & 1050 & 1050 \\
\hline$\left(\mathbf{C}_{1}\right)$ & Net benefit (LE) & 2052.8 & 897.2 & 1628.4 & 1495.2 & 1729.2 \\
\hline & Mean of grain yield $\left(\mathrm{ard} / \mathrm{fed}^{-1}\right)$ & 8.77 & 5.87 & 7.70 & 7.36 & 8.17 \\
\hline Giza & Gross benefit (LE) & 3139.2 & 2109.6 & 2772 & 2631.6 & 2941.2 \\
\hline 134 & Costs that vary (L.E) & 1000 & 1000 & 1050 & 1050 & 1050 \\
\hline$\left(\mathbf{C}_{2}\right)$ & Net benefit (LE) & 2139.2 & 1109.6 & 1722 & 1621.6 & 1891.2 \\
\hline \multirow{5}{*}{$\begin{array}{l}\text { Giza } \\
2000 \\
\left(\mathbf{C}_{3}\right)\end{array}$} & Mean of grain yield $\left(\mathrm{ard} / \mathrm{fed}^{-1}\right)$ & 9.69 & 6.37 & 8.58 & 8.39 & 9.21 \\
\hline & Gross benefit (L E) & 3488.4 & 2293.2 & 3085.2 & 2952 & 3283.2 \\
\hline & Costs that vary (LE) & 1000 & 1000 & 1050 & 1050 & 1050 \\
\hline & Net benefit (LE) & 2488.4 & 1293.2 & 2035.2 & 1902 & 2233.2 \\
\hline & \multicolumn{6}{|c|}{ Green forage yield $\left(\right.$ Ton$/$ fed $\left.^{-1}\right)$} \\
\hline & Mean of green forage yield (ton/fed $\left.{ }^{-1}\right)$ & - & 5.14 & 5.38 & 5.31 & 5.50 \\
\hline Giza & Gross benefit (LE) & - & 1551.0 & 1593 & 1518 & 1638 \\
\hline 133 & Costs that vary (LE) & - & 300 & 300 & 300 & 300 \\
\hline$\left(\mathbf{C}_{1}\right)$ & Net benefit (L E) & - & 1251.0 & 1293 & 1278 & 1338 \\
\hline & Mean of green forage yield (ton/fed $\left.{ }^{-1}\right)$ & - & 5.71 & 5.88 & 5.75 & 5.95 \\
\hline Giza & Gross benefit (LE) & - & 1698 & 1755 & 1719 & 1788 \\
\hline 134 & Costs that vary (LE) & - & 300 & 300 & 300 & 300 \\
\hline$\left(\mathbf{C}_{2}\right)$ & Net benefit (LE) & - & 1398 & 1455 & 1419 & 1488 \\
\hline Giza & Mean of green forage yield (ton/fed $\left.{ }^{-1}\right)$ & - & 5.87 & 6.72 & 6.48 & 6.87 \\
\hline 2000 & Gross benefit (LE) & - & 2025 & 2067 & 2055 & 2097 \\
\hline$\left(\mathbf{C}_{3}\right)$ & Costs that vary (LE) & - & 300 & 300 & 300 & 300 \\
\hline & Net benefit (LE) & - & 1725 & 1767 & 1755 & 1797 \\
\hline \multirow{3}{*}{\multicolumn{2}{|c|}{$\begin{array}{l}\text { *Difference in net benefit for } \mathrm{C}_{1} \text { (grain-green) } \mathrm{LE} \\
\text { Difference in net benefit for } \mathrm{C}_{2} \text { (grain-green) } \mathrm{LE} \\
\text { Difference in net benefit for } \mathrm{C}_{3} \text { (grain-green) } \mathrm{LE}\end{array}$}} & 2052.8 & 353.8 & 335.4 & 217.2 & 391.2 \\
\hline & & 2139.2 & 288.4 & 267 & 202.6 & 403.2 \\
\hline & & 2488.4 & 431.8 & 268.2 & 147 & 436.2 \\
\hline \multirow{3}{*}{\multicolumn{2}{|c|}{$\begin{array}{l}\text { *Difference in the cost that vary for } C_{1} \text { (grain-green) } \mathrm{LE} \\
\text { Difference in the cost that vary for } C_{2} \text { (grain-green) } \mathrm{LE} \\
\text { Difference in the cost that vary for } C_{3} \text { (grain-green) } \mathrm{LE}\end{array}$}} & 1000 & 700 & 750 & 750 & 750 \\
\hline & & 1000 & 700 & 750 & 750 & 750 \\
\hline & & 1000 & 700 & 750 & 750 & 750 \\
\hline \multirow{3}{*}{\multicolumn{2}{|c|}{$\begin{array}{l}\text { *Total benefit for } \mathrm{C}_{1} \text { (grain+ green) } \mathrm{LE} \\
\text { Total benefit for } \mathrm{C}_{2} \text { (grain+ green) } \mathrm{LE} \\
\text { Total benefit for } \mathrm{C}_{3} \text { (grain+ green) } \mathrm{LE}\end{array}$}} & 2052.8 & 2148.2 & 2921.4 & 2773.2 & 3067.2 \\
\hline & & 2139.2 & 2507.6 & 3177 & 3040.6 & 3379.2 \\
\hline & & 2488.4 & 3018.2 & 3802.2 & 3657.0 & 4030.2 \\
\hline \multicolumn{2}{|c|}{$* M R R \%$ for $\mathrm{C}_{1}$} & - & 50.54 & 51.54 & 33.41 & 60.1 \\
\hline \multicolumn{2}{|c|}{ MRR $\%$ for $C_{2}$} & - & 41.2 & 41.08 & 31.16 & 62.30 \\
\hline \multicolumn{2}{|c|}{ MRR \% for $C_{3}$} & - & 61.69 & 89.26 & 22.61 & 67.11 \\
\hline \multirow{2}{*}{\multicolumn{2}{|c|}{ L. S. D at 0.05 for $\mathrm{C} x \mathrm{~T}$}} & \multicolumn{2}{|c|}{$C_{1}=2.69$} & $\begin{array}{l}\mathrm{C}_{2}= \\
2.05\end{array}$ & \multicolumn{2}{|c|}{$\mathrm{C}_{3}=2.16$} \\
\hline & & \multicolumn{5}{|c|}{$\mathbf{0 . 3 3}$} \\
\hline \multicolumn{2}{|c|}{$\begin{array}{l}\text { *Difference in the cost LE pound = (costs that vary for grain yield L.E. - costs that vary for grain yield LE). } \\
\text { *Total benefit LE }=(\text { net benefit for grain yield L.E. + net benefit for green forage yield LE }) . \\
\text { *MRR } \%=(\text { differences in NB/differences in costs that vary) X } 100 .\end{array}$} & \multicolumn{4}{|c|}{$\begin{array}{l}* \mathrm{~T}_{1}=\text { cut plants without spray IAA, } \mathrm{T}_{2}=\text { cut plants and foliar spray of IAA at } 20 \text { days after cut, } \mathrm{T}_{3}=\text { cut plants and foliar spray } \\
\text { of IAA at } 40 \text { days and } \mathrm{T}_{4}=\text { cut plants and foliar spray of IAA twice at } 20 \text { and } 40 \text { days after cut using. } \\
* \text { Difference in net benefit LE pound= (grain yield L.E. - green forage yield LE). }\end{array}$} & pray \\
\hline
\end{tabular}

\section{Conclusion}

When barley was grown for forage and grain yield, IAA can be used as foliar spray at early time after forage cutting to enhance plants recovery.

\section{REFERENCES}

Abdel-Aziz El-Set A., Abdel-Gawad M. H. and
El-Batal M. A. (2004). Effect of foliar spray with IAA after cutting plants on growth and yield of barley. Egypt. J. Appl. Sci., 19 (6B).

Abdo Fatma A. and Abdel-Aziz El-Set A. (2009). Response of soybean to foliar spraying with growth regulators mixture and zinc. Egypt. J. Appl. Sci. 24: 215-238. 
Al-Satari Y., A., Kafawin O., Ghawi I. and Saoub H. M. (2001). Response of two barley cultivars to three seeding rates under supplemental irrigation. Arab-Gulf J. Sci. Res., 19 (1): 7-11.

Angela K. S., and Gray W. M. (2011). Plant hormone receptors: new perceptions. Genes \& dev., 22: 2139-2148.

A.O.A.C, (2000). Association of Official Agricultural Chemists. Official Methods of Analysis $17^{\text {th }}$ ed., AOAC., Washington DC, USA.

Barsoum M. S. (1994). Response of barley to IAA presoaking grain at South Sinai. Ann. Agric. Sci., Moshtohor, Egypt. 32 (3): 1355-1369.

Baz A. I. O., Safwat M. S. A. and Abdallah A. R. (1984). Some physiological studies on soybean plants: I. Effect of some growth regulators on growth, yield, modulation and chemical composition. Ann. Agric. Sci. 21: 479- 494.

CIMMYT (1988). From Agronomic Data to Farmer analysis of stevia (Stevia rebaudiana Bert.) cultivation through stem cutting and tissue culture propagule in India. Trends Agric., Econ., 3: 216- 222.

El-Bawab A. M. O. and Sandak R. N. (2002). Agronomical and biochemical evaluation for some exotic barley genotypes. Egypt. J. Agric. Res., 80 (2): 817-834.

Govindan K. and Thirumurugan V. S. (2000). Response of soybean to growth regulators. Res. Crops Ind., 1: 323-325.

Harsharn S. and Gill H. S. (1985). Effect of foliar spray of NAA on the growth and yield of late sown wheat and barley. Indian J. Ecol., 12 (2): 267-272.

Kalarani M. K. and Jeyakumar P. (1998). Effect of nutrient and NAA spray on physiological changes in soybean (Glycine $\max$ L.). Indian J. Plant Physiol., 3: 226228.

Mona E. Eleiwa, Maymona A. Kord and Ibrahim S. A. (2013). Response of barley plants to foliar application of growth regulators mixture of indole acetic acid and zinc. Afr. J. Biotec., 12 (23): 3653-3661.

Royo C. (1999). Plant recovery and grain yield formation in barley and triticale following forage removal at two cutting stages. J. Agron. and Crop. Sci., 182 (3): 175-183.

Royo C. and Tribo F. (1997). Tritticale and barley for grain and for dual-purpose (forage + grain) in a Mediterranean type environment. I. Growth analysis. Aust. J. Agric. Res., 48 (4): 411-421.

Salem H. M. (1990). Physiological response of barley plant to $\mathrm{GA}_{3}$ and IAA applied as grain treatment. Annals Agric. Sci., Moshtohor, Egypt, 28 (4): 1975-1984.

Senthil A. Pathmanaban G. and Srinivasan P.S. (2003). Effect bioregulators on some physiological and biochemical parameters of soybean (Glycine max L.). Legume Res. India, 26: 54-56.

Sharma N. K. (1998). Note on forage productivity of oat and barley with different growth regulators. Current Agric., India, 22 (1-2): 125-126.

Sharma N. K. (2002). Relative performance of oat and barley cultivars for forage yield. Forage Res., India, 28 (2): 113-114.

Snedecor George W. and Cochran William G. (1989). Statistical Methods, $8^{\text {th }}$ Ed., Iowa State University Press, USA.

Steel R. G. D. and Torrie J. H. (1980). Principles and procedures of statististics. Mc-Graw Hill Co.(pub.), Singapore. $2^{\text {nd }}$ Ed., $4^{\text {th }}$ Prin., 633 pp.

Strickler F. C. (1964). Row width \& plant population studies with corn. Agron, J. 56: 438-441.

Tiwari D. K., Pandey P., Giri S. P. and Dwivedi J. L. (2011). The exogenous application of various plant growth hormones on grain yield. Asian. J. Plant Sci. 10: 133-139.

Velu G. (1999). Impact of soil moisture stress and ameliorants on growth of soybean. Madras Agric. J., India, 36: 330-332.

Witham F. H., Blaydes D. F. and Devlins R. M. (1971). Experiments in plant physiology. Van Nostrand Reinhold, (pub.) New York, USA.

Yau S. K. (1999). Sustaining barley yield by early planting and grazing. Rachis, 18 (2): 72-74.

Yau S. and Yau S. K. (2003). Yields of early planted barley after clipping or grazing in a semiarid area. Agron. J., 95 (4): 821827.

Zaki R. N. and Radwan T. E. (2011). Improving wheat grain yield and it's quality under salinity conditions at a newly reclaimed soil using different organic sources. J. App. Sci. Res., 7: 42-55. 


\section{تأثير الرش بأندول حمض الخليك على النمو و محصول الحبوب لبعض أصناف الثعير الحديثة بعد الحش فى الأراضى الجديدة فى الأسماعلية \\ * محمد زكريا محمود شندى - محمود احمد مدكور}

قسم بحوث الثعير و *قسم بحوث فسيولوجيا المحاصيل - معهد بحوث المحاصيل الحقلية

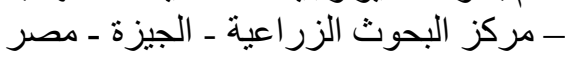

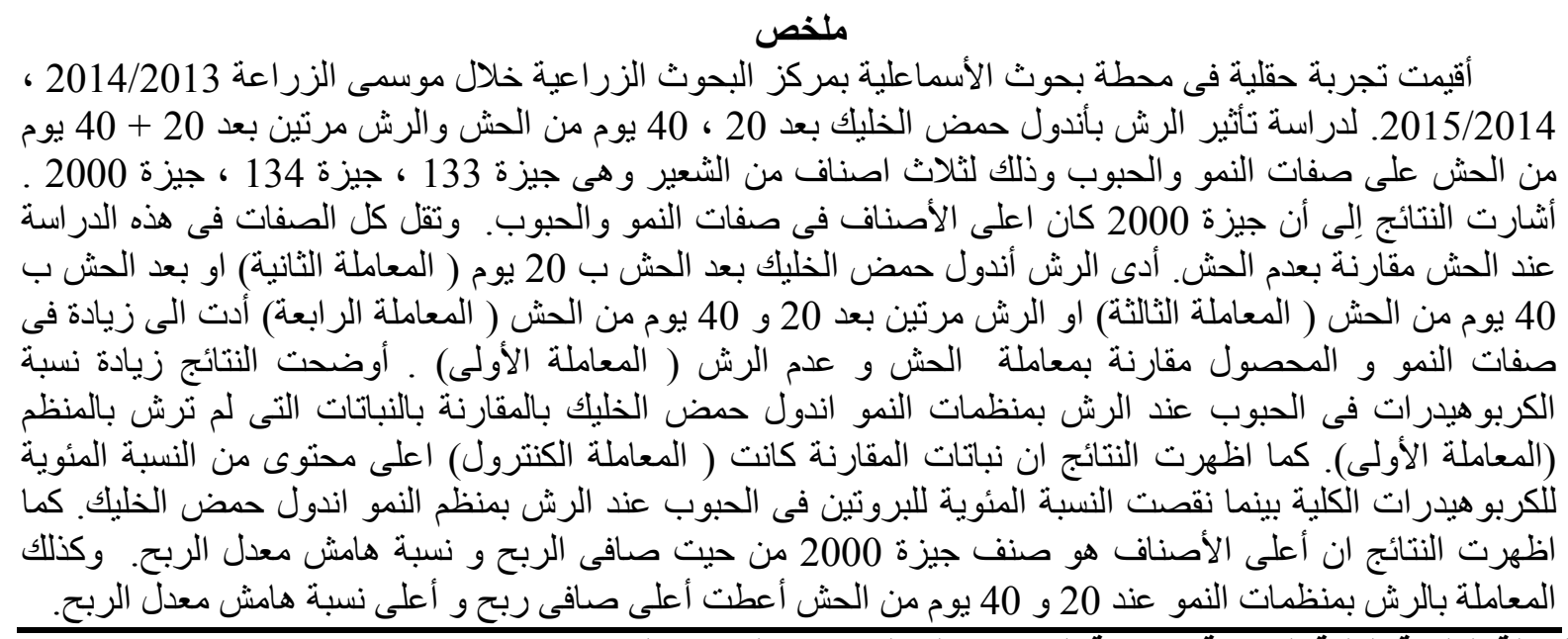

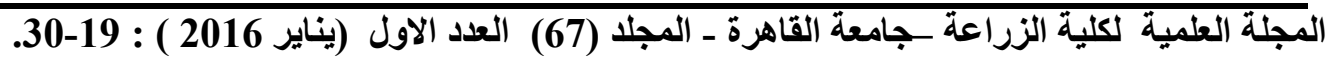

